\title{
Differential Methylation of Syncytin-1 and 2 Distinguishes Fetal Growth Restriction from Physiologic Small for Gestational Age
}

\author{
Sami P. Makaroun, MD ${ }^{1,2}$ Katherine P. Himes, MD, MS $S^{1,2}$ \\ 1 Division of Maternal Fetal Medicine, Magee-Womens Research \\ Institute, University of Pittsburgh, Pittsburgh, Pennsylvania \\ 2 Department of Obstetrics, Gynecology and Reproductive Sciences, \\ University of Pittsburgh, School of Medicine, Pittsburgh, \\ Pennsylvania \\ Address for correspondence Sami P. Makaroun, MD, Department of \\ Obstetrics, Gynecology and Reproductive Sciences, University of \\ Pittsburgh School of Medicine, 300 Halket Street, Pittsburgh, \\ PA 15213 (e-mail: makarounsp2@upmc.edu).
}

Am J Perinatol Rep 2018;8:e18-e24.

\begin{abstract}
Objective The retroviral genes encoding Syncytin-1 (SYN1) and Syncytin-2 (SYN2) are epigenetically regulated, uniquely expressed in the placenta and critical to placental function. We sought to determine if placental expression and methylation patterns of SYN1 and SYN2 from pregnancies complicated by fetal growth restriction (FGR) differed from physiologic small for gestational age (SGA) and appropriate for gestational age (AGA) controls.

Study Design Placental biopsies were obtained from AGA, SGA and FGR neonates delivered at $>36$ weeks gestation. SGA and FGR were defined as birth weight $<10 \%$ with

Keywords

- retroelements

- syncytin

- methylation

- placenta

- fetal growth restriction

- intrauterine growth restriction FGR additionally requiring abnormal fetal testing. We quantified DNA methylation of SYN1 and SYN2 by EpiTyper and gene expression by RT-qPCR.

Results We identified 10 AGA, 9 SGA and 7 FGR placentas. There was decreased methylation in SYN1 and SYN2 in FGR relative to AGA and SGA. When the sum of SYN1 and SYN2 methylation was used for prediction of FGR from SGA, the area under the receiver operator characteristic curve was $0.9048(0.7602,1)$.

Conclusion SYN1 and SYN2 methylation marks differ in FGR and SGA. We plan future studies to examine these markers in cell free DNA to determine if these methylation changes could be used as a biomarker for FGR.
\end{abstract}

Fetal growth restriction (FGR), conventionally defined as an ultrasound estimated fetal weight less than the 10th centile, is associated with an increased risk of perinatal morbidity and mortality as well as an increased risk of adult diseases. ${ }^{1}$ Many fetuses identified as less than the 10th centile, however, are not pathologically small, but rather constitutionally small for gestational age (SGA). Additionally, due to the inherent error of ultrasound estimation of fetal weight, which may be as great as $25 \%$, fetuses that are appropriate for gestational age (AGA) can be misclassified as FGR. ${ }^{2}$ Constitutionally SGA and misclassified AGA fetuses are not at higher risk of antenatal or

received

August 26, 2016

accepted after revision

December 9, 2017
DOI https://doi.org/

$10.1055 / \mathrm{s}-0038-1627473$ ISSN 2157-6998. postnatal complications. They are, however, subject to the cost and morbidity of invasive testing, antenatal monitoring, and iatrogenic preterm delivery. Given this, identifying potential biomarkers that can distinguish these groups of fetuses would have clinical and cost benefits.

Transposable elements are a class of mobile genetic elements that have been estimated to comprise half of the human genome. ${ }^{3}$ Retroelements (REs), a subset of transposable elements, originate from retroviruses, integrate into the germline and are thus transmitted to all the cells of the host. ${ }^{3}$ REs can cause insertional mutagenesis or other adverse effects and are
Copyright $\odot 2018$ by Thieme Medical Publishers, Inc., 333 Seventh Avenue, New York, NY 10001, USA. Tel: +1(212) 584-4662.
License terms

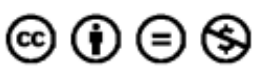


often suppressed in somatic tissues by epigenetic modifications, including DNA methylation. ${ }^{4}$

Interestingly, REs are often hypomethylated and highly expressed in the placenta. Furthermore, some REs have been co-opted to perform essential functions in the placenta., 6 For example, Syncytin-1, encoded by ERVW-1 (SYN1), and Syncytin-2, encoded by ERVFRD-1 (SYN2), have intact env genes that have evolved to mediate cell-to-cell fusion in the placenta to form the syncytiotrophoblast. ${ }^{7}$ Mice that lack expression of Syncytin-A (the murine orthologue of Syncytin-1), die between 11.5 and 13.5 days of gestation due to failure of the syncytial layer to form. ${ }^{8,9}$ Limited studies from human pregnancy suggest these gene products are also important in the human placenta. Altered expression and methylation patterns are associated with growth discordance in twin pregnancies and FGR or other placental syndromes in singleton pregnancies. ${ }^{10-14}$ As a mutable epigenetic mark, methylation patterns may be of particular interest because they are more likely than fixed genetic marks to reflect the environmental circumstances that may predispose to FGR and other placental syndromes.

Given the importance of Syn 1 and Syn 2 to placental function, our primary objective was to determine if methylation and expression patterns of SYN1 and SYN2 differed from FGR placentas compared with SGA placentas. We hypothesized that expression or methylation differences in Syncytin -1 and Syncytin-2, would plausibly distinguish pathologic FGR from constitutional SGA.

\section{Materials and Methods}

\section{Placental Biopsies}

Samples were obtained from a placental biopsy biobank that is maintained at the Magee-Womens Research Institute. A trained research nurse obtained at least two placental biopsy samples immediately after delivery. One sample was snap frozen in liquid nitrogen and the other was placed in RNAlater (Qiagen, Hilden, Germany) and stored at $-80^{\circ} \mathrm{C}$. A chart abstraction was performed at the time of collection and entered into a de-identified database linked to the samples. The University of Pittsburgh Institutional Review Board under project number PRO08050177 approved specimen collection.

\section{Subject Selection Criteria}

Using the Magee Biobank database, placental biopsy samples were selected from singleton pregnancies delivered after 36 weeks gestation. Women with diabetes mellitus or those carrying fetuses with suspected anomalies or aneuploidy were excluded. For analysis, subjects were divided into AGA $(n=10)$, SGA $(n=9)$, and FGR $(n=7)$ groups. AGA was defined by birth weight $>10$ th centile and $<90$ th centile using the Alexander growth reference. ${ }^{15}$ Subjects in both the SGA and FGR groups had birth weight $\leq 10$ th centile. Subjects that were categorized in the FGR group had antenatal evidence of uteroplacental insufficiency, defined as oligohydramnios, decreased fetal movement, or abnormalities in the biophysical profile, nonstress testing, contraction stress testing, or umbilical artery Doppler waveform.

\section{Identifying Candidate Genes}

The main target genes for this study were SYN1 and SYN2. To explore how SYN1 and SYN2 compare with other REs present in the placenta, we identified other REs for analysis that are expressed in the placenta or have placental-specific RE-derived regulatory regions. REs in the former category included endogenous retrovirus group 3(ERV-3), paternally expressed 10 (PEG10) and retrotransposon-like 1(RTL1), while the latter included leptin $(L E P)$, endothelin receptor $\mathrm{B}(E D N R B)$, aromatase (CYP19A1), early placenta insulin-like peptide(INSL4), midline-1(MID1), and pleiotrophin $(P T N) .{ }^{5}$ Expression analysis was performed in all of these and methylation assessment performed on SYN1, SYN2, PEG10, and PTN.

\section{Real-time Quantitative Polymerase Chain Reaction}

Each sample was mechanically homogenized and digested in TRIzol followed by chloroform extraction and 100\% ethanol precipitation. RNAwas transferred to silica spin-columns (Epoch Life Science, Missouri City, TX) for on column RNase-free DNase treatment (Qiagen, Hilden, Germany) and washing. The RNA pellet was suspended in RNase-free water. Quantification and purity testing of the eluted RNA was performed by spectrophotometric analysis at $\mathrm{OD}_{260}$ and $\mathrm{OD}_{280}$ with the NanoDrop 1000 and by gel electrophoresis. Samples of 8 AGA, 6 SGA, and 4 FGR had high quality RNA for expression analysis. Complementary DNA was prepared using Applied Biosystems' high-capacity RNA-to-cDNA ${ }^{\mathrm{TM}}$ kit (Thermo Scientific, Waltham, MA) per the manufacturer's instructions. Primers were identified using the Massachusetts General Hospital Primer Bank (https://pga.mgh. harvard.edu/primerbank/index.html) and checked for specificity using the National Center for Biotechnology Information's Primer BLAST (http://www.ncbi.nlm.nih.gov/tools/primer-blast /index.cgi?LINK_LOC=BlastHome). The primer sequences used are listed in - Table 1. RT-qPCR was performed in triplicate using SYBR ${ }^{\circledR}$ green PCR Master Mix (ThermoScientfic, Waltham, MA) and the ViiA ${ }^{\text {TM }} 7$ Real-Time PCR System (ThermoScientfic, Waltham, MA). A template control was run for each primer set and samples were analyzed using the DDCt method (delta-delta cycle threshold). YWHAZ was used as the internal control.

\section{DNA Extraction}

Each sample was mechanically homogenized and placed in DNA digest buffer with Proteinase $\mathrm{K}$ at $50^{\circ} \mathrm{C}$ for 3 hours. RNase A was added and a 1:1 phenol/chloroform extraction subsequently performed. The samples were washed with chloroform and DNA was precipitated using $100 \%$ ethanol with a $70 \%$ ethanol wash. The resulting pellet was suspended in TE buffer. Quantification and purity testing of DNA was performed with spectrophotometric analysis at $\mathrm{OD}_{260}$ and $\mathrm{OD}_{280}$ with the NanoDrop 1000 (ThermoScientific, Waltham, MA).

\section{DNA Methylation}

The region of interest for methylation assessment was identified based on previous studies showing methylation changes in regulatory regions for each gene. ${ }^{14,16-20}$ The final determined base positions, based on the Genome Reference Consortium build 38, were 92477267-92478260 on chromosome 7 for SYN1, 11111566-11112154 on chromosome 6 
Table 1 RT-qPCR primers used for expression studies

\begin{tabular}{|c|c|c|}
\hline Gene & Primer pair sequences & $\begin{array}{l}\text { Amplicon } \\
\text { size } \\
\text { (base pairs) }\end{array}$ \\
\hline \multirow[t]{2}{*}{ SYN1 } & f GAAGGCCCTTCATAACCAATGA & \multirow[t]{2}{*}{83} \\
\hline & r GATATTTGGCTAAGGAGGTGATGTC & \\
\hline \multirow[t]{2}{*}{ SYN2 } & f TACACCCACAACCAATTCCGC & \multirow[t]{2}{*}{93} \\
\hline & r CCGGCTGGATTTATCTAGCAAAG & \\
\hline \multirow[t]{2}{*}{ ERV3 } & f TGTTCTTGСТАСТССССТTATCC & \multirow[t]{2}{*}{86} \\
\hline & r GTTCCCCGACCACGTAGTG & \\
\hline \multirow[t]{2}{*}{ PEG10 } & f AACGCAAGATCAGACGCCTG & \multirow[t]{2}{*}{75} \\
\hline & r GGGCAATCATCTGGAAAGCAT & \\
\hline \multirow[t]{2}{*}{$R T L$} & f GTCATGCAACGGTTCACACC & \multirow[t]{2}{*}{86} \\
\hline & r CCGATGGGTTGACTGATGCT & \\
\hline \multirow[t]{2}{*}{$L E P$} & f GACACTGGCAGTCTACCAACAGAT & \multirow[t]{2}{*}{97} \\
\hline & r GTGAAGAAGATCCCGGAGGTT & \\
\hline \multirow[t]{2}{*}{ CYP19A1 } & f CCACAGCTGAGAAACTGGAAGA & \multirow[t]{2}{*}{78} \\
\hline & r TCGTCAGGTCTCCACGTCTCT & \\
\hline \multirow[t]{2}{*}{ EDNRB } & f GGGAAGGAACTGGTACTTGG & \multirow[t]{2}{*}{110} \\
\hline & r ACTTGGAGGCGGCTGCATG & \\
\hline \multirow[t]{2}{*}{ INSL4 } & f AGCCTGTTCCGGTCCTATCT & \multirow[t]{2}{*}{211} \\
\hline & r TGTTGGAGGTTGACACCATTTC & \\
\hline \multirow[t]{2}{*}{ MID1 } & f СTGACСTGCССТАTTTGTСTG & \multirow[t]{2}{*}{107} \\
\hline & r GCACAGTGTGATACTAGGATGC & \\
\hline \multirow[t]{2}{*}{ PTN } & f GGAGCTGAGTGCAAGCAAAC & \multirow[t]{2}{*}{157} \\
\hline & r CTCGCTTCAGACTTCCAGTTC & \\
\hline
\end{tabular}

for SYN2, 94656185-94656702 on chromosome 7 for PEG10, and 137268077-137268597 on chromosome 7 for PTN. Genomic DNA methylation patterns were determined by EpiTYPER application (Agena Bioscience, San Diego, CA) as previously described ${ }^{21}$ (Roswell Park Cancer Institute Genomics Shared Resource with Core grant NCI P30CA16056, Buffalo, NY). Three amplicons were needed for SYN1and SYN2 and two amplicons for PEG10 and PTN. Samples were run in duplicate. Each amplicon was analyzed separately using mean CpG methylation. Only differentially methylated regions are presented in the results. These regions are amplicon 2 for SYN1, amplicon 1 for SYN2, and amplicon 1 for PEG10. The primer sequences used are listed in - Table 2.

\section{Statistical Analysis}

Given the distribution of the data, Kruskal-Wallis test was used to compare median expression fold change and methylation levels between all three groups. If significant, two-way comparisons were then performed with the Wilcoxon ranksum test and reported in the results. The summation of SYN1 and SYN2 methylation percentages in differentially methylated amplicons was then analyzed as a possible predictive test. Receiver operating characteristic curves were generated and sensitivity and specificity were calculated. Categorical baseline data were analyzed by chi-square testing. Statistical significance was defined by $p$-value $<0.05$ in all analyses.

\section{Results}

\section{Patient Characteristics}

A total of 26 specimens were identified from the placenta biobank-10 AGA, 9 SGA, and 7 FGR. As expected, these groups differed significantly by gestational age at birth, placental and birth weights, mode of delivery, and performance of umbilical artery cord gas (-Table 3). Specifically, FGR babies weighed $470 \mathrm{~g}$ less than SGA and $690 \mathrm{~g}$ less than AGA babies at birth ( $p<0.001)$. Only $43 \%$ of FGR babies were delivered vaginally, compared with $100 \%$ of SGA babies and $90 \%$ of AGA babies $(p=0.01)$.

\section{SYN1 Expression is Significantly Increased in Both FGR and SGA Placentas}

Placental SYN1 expression was significantly increased in both FGR and SGA samples compared to AGA samples ( $p=0.027$ and $p=0.005$, respectively). There was, however, no significant difference between SYN1 expression in SGA and FGR placenta. There was no difference in SYN2 expression between the three groups (-Fig. 1A and B).

\section{SYN1 and SYN2 Methylation is Decreased Uniquely in FGR Placentas}

Methylation of SYN1 was decreased in FGR samples [23.5\% CpG methylation (IQR 21.5, 26.5)] compared with SGA [29.6\% CPG methylation (IQR 24.0, 32.1); $p=0.044]$ and AGA [28.9\% CpG methylation (IQR 26.6, 33.5); $p=0.006$ ]. Interestingly, despite the lack of change in expression patterns, SYN2 methylation was also decreased in FGR samples [16.5\% CpG methylation (IQR 14.8, 19.8)] compared with SGA [21.9\% CpG methylation (IQR 19.8, 22.3); $p=0.008$ ] and AGA [22.9\% CpG methylation (IQR 21.7, 24.0); $p=0.011]$ (-Fig. 1C and D).

\section{SYN1 and SYN2 Methylation Accurately Identifies FGR}

Methylation for SYN1 $<27 \%$ and SYN2 $<21 \%$ had a sensitivity of $100 \%$ and specificity of $66.7 \%$ for distinguishing FGR from SGA in this cohort. The receiver operating characteristic (ROC) curve (-Fig. 2) generated for the sum of these methylation percentages used for prediction of FGR from SGA had an area under the curve of 0.9048 (95\% confidence interval [CI] $0.7602-1$ ) with a possible $100 \%$ sensitivity and $66.7 \%$ specificity. When used to distinguish FGR from both SGA and AGA, the generated ROC curve had an area under the curve of $0.9474(95 \%$ CI $0.8674-1)$ with a possible $100 \%$ sensitivity and $79 \%$ specificity.

\section{The Methylation Pattern in SYN1 and SYN2 is Unique to These Retroelements}

There was no statistical difference between the three groups in expression in other REs that are highly expressed in the placenta including ERV3, PEG10, RTL1, LEP, EDNRB, CYP19A1, INSL4, MID1 or PTN. There was a trend toward increased expression in FGR samples in PEG10 [1.15 fold change for 
Table 2 Bisulfite primers used for methylation studies

\begin{tabular}{|c|c|c|c|}
\hline Amplicon & Bisulfite primer pair sequences & Genomic location $^{a}$ & CpGs \\
\hline \multirow[t]{2}{*}{ SYN1 Amp 1} & f TAGGATTTAGAGGGATGGGAGTTAG & \multirow[t]{2}{*}{ Chr 7: 92477756-92478001 } & \multirow[t]{2}{*}{7} \\
\hline & r AACACAACAAAAAAAACAACAATC & & \\
\hline \multirow[t]{2}{*}{ SYN1 Amp 2} & f TAAGGAATGGAATTTTGGGTTATGT & \multirow[t]{2}{*}{ Chr 7: $92477545-92477776$} & \multirow[t]{2}{*}{6} \\
\hline & r СТСССАТСССТСТАААТССТАСAA & & \\
\hline \multirow[t]{2}{*}{ SYN1 Amp 3} & f TTTTAATTTTAAGGAAGGATAGGATAGA & \multirow[t]{2}{*}{ Chr 7: 92477322-92477531 } & \multirow[t]{2}{*}{5} \\
\hline & r CAAAAACTCCAAATCAAAAAATAC & & \\
\hline \multirow[t]{2}{*}{ SYN2 Amp 1} & f GGGGTGAGTAGAGAGAGTAGTTAGGG & \multirow[t]{2}{*}{ Chr 6: 11111515-11111750 } & \multirow[t]{2}{*}{8} \\
\hline & r AACCCCAAATCAAAAACTAAACAAA & & \\
\hline \multirow[t]{2}{*}{ SYN2 Amp 2} & f TGGTTTGTTAGTATTTGGGAGGAGT & \multirow[t]{2}{*}{ Chr 6: 11111831-11112179 } & \multirow[t]{2}{*}{6} \\
\hline & r AAAAAAACCCCCAACTCAAAAATAT & & \\
\hline \multirow[t]{2}{*}{ SYN2 Amp 3} & f TGTTTTATTATTAGGGAAGGTATT & \multirow[t]{2}{*}{ Chr 6: 11111669-11111908 } & \multirow[t]{2}{*}{4} \\
\hline & r AAAAAATATCTCAAAAAAACATAC & & \\
\hline \multirow[t]{2}{*}{ PEG10 Amp 1} & f TAGGGGTTTTTTAGTTTTTAATTAT & \multirow[t]{2}{*}{ Chr 7: 94656061-94656355 } & \multirow[t]{2}{*}{17} \\
\hline & r CTATAAACCTTATATAAATTACACC & & \\
\hline \multirow[t]{2}{*}{ PEG10 Amp 2} & f GGTGTAATTTATATAAGGTTTATAGTTT & \multirow[t]{2}{*}{ Chr 7: $94656331-94656672$} & \multirow[t]{2}{*}{26} \\
\hline & r TTCTAAAATACTACTCCATCTCCC & & \\
\hline \multirow[t]{2}{*}{ PTN Amp 1} & f TGTTGATGTTTTTAGTTGATTAAAGTTA & \multirow[t]{2}{*}{ Chr 7: c137268427-137268055 } & \multirow[t]{2}{*}{24} \\
\hline & r ACAAATTCCAAAAACTAATCTTACC & & \\
\hline \multirow[t]{2}{*}{ PTN Amp 2} & f TGAAATTAGGTTTGGGTTTGTTTG & \multirow[t]{2}{*}{ Chr 7: c137268719-137268452 } & \multirow[t]{2}{*}{6} \\
\hline & r CCTCAAATACTCAACTTCTATCCСТTTT & & \\
\hline
\end{tabular}

Abbreviations: Amp, amplicon; Chr, chromosome.

${ }^{a}$ Genomic location based on Genome Reference Consortium build 38.

Table 3 Baseline characteristics by group

\begin{tabular}{|l|l|l|l|l|}
\hline & AGA $(\boldsymbol{n}=\mathbf{1 0})$ & SGA $(\boldsymbol{n}=9)$ & FGR $(\boldsymbol{n}=7)$ & $p$-value \\
\hline Gestational age, weeks & 37.4 & 37.9 & 36.8 & 0.037 \\
\hline Birthweight, grams & 3,052 & 2,362 & 1,892 & $<0.001$ \\
\hline Placental weight, grams & 624 & 367 & 302 & $<0.001$ \\
\hline Cord gas done, $n(\%)$ & $0(0)$ & $3(33)$ & $6(86)$ & 0.001 \\
\hline Umbilical artery pH & NA & 7.26 & 7.20 & 0.365 \\
\hline Induced, $n(\%)$ & $1(10)$ & $8(89)$ & $6(86)$ & 0.001 \\
\hline Vaginal delivery, $n(\%)$ & $9(90)$ & $9(100)$ & $3(43)$ & 0.010 \\
\hline Female fetus, $n(\%)$ & $7(30)$ & $1(11)$ & $5(71)$ & $1(14)$ \\
\hline Maternal smoking, $n(\%)$ & $0(0)$ & & 0.075 \\
\hline
\end{tabular}

Abbreviations: AGA, appropriate for gestational age; FGR, fetal growth restriction; SGA, small for gestational age.

AGA (IQR 0.95, 1.36) vs. 1.59 fold change for SGA (IQR 1.26, $2.85)$ vs. 2.31 fold change for FGR (IQR 1.14, 3.33)] and PTN [0.66 fold change for AGA (IQR 0.38, 0.83) vs. 1.20 fold change for SGA (IQR 0.81, 1.76) vs. 1.02 fold change for FGR (IQR 0.58, 1.38)], and thus methylation analyses for these genes were performed. In contrast to SYN1 and SYN2, the differentially methylated region of PEG10 showed higher methylation in FGR [50.2\% CpG methylation (IQR 43.1, 60.2)] compared with AGA [21.0\% CpG methylation(IQR 11.1, 23.8); $p=0.005$ ], but not to
SGA samples [41.8\% CpG methylation (IQR 29.0, 42.4)]. Including this with SYN1 and SYN2 methylation did not improve the area under the curve of the generated ROC curves, so was not considered in the final predictive model above.

\section{Discussion}

The current study demonstrates significantly lower placental methylation of the regulatory regions of SYN1 and SYN2 in 


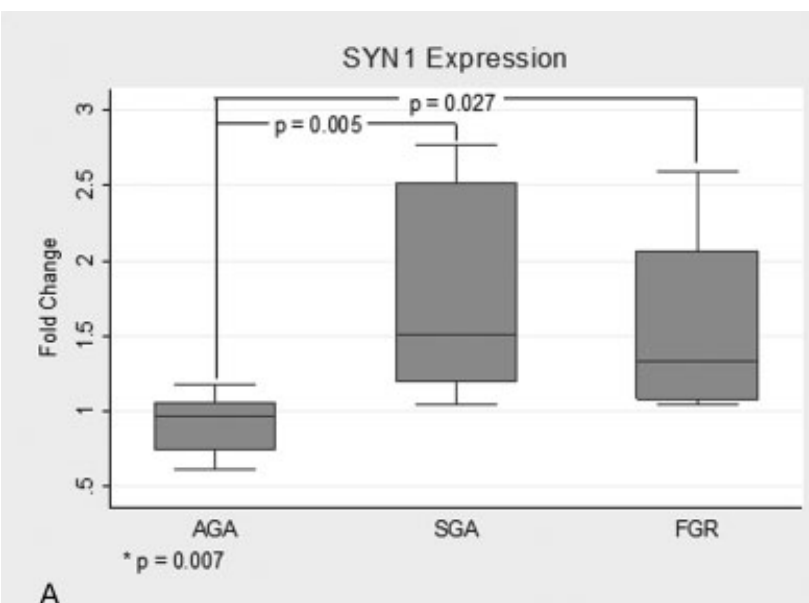

A

SYN1 Methylation
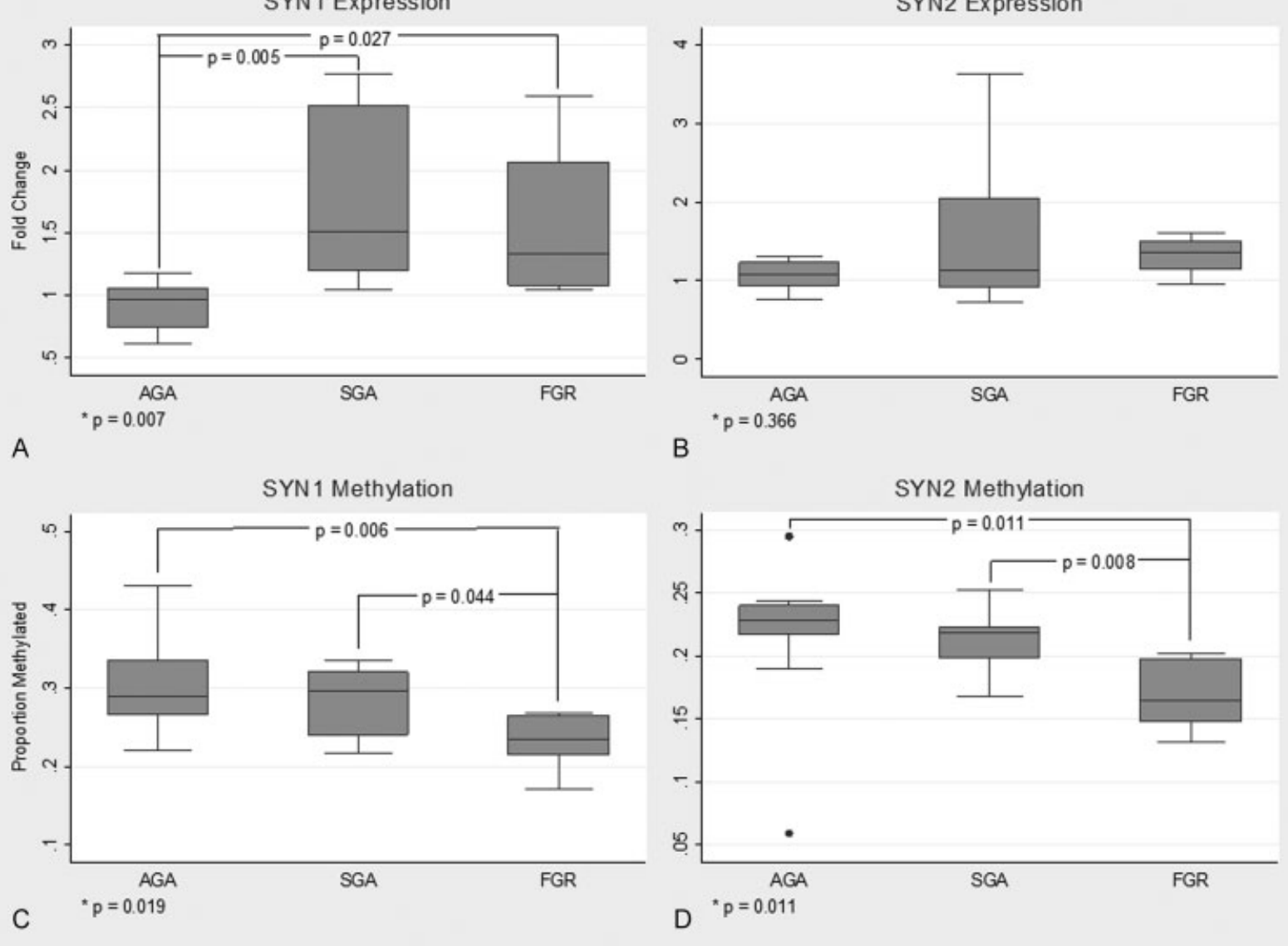

Fig. 1 Expression and DNA methylation of SYN1 and SYN2 by group. (A) SYN1 expression, (B) SYN2 expression, (C) SYN1 methylation, and (D) SYN2 methylation. * Three-way comparisons for $p$-values using the Kruskal-Wallis test. AGA, appropriate for gestational age; FGR, fetal growth restriction; SGA, small for gestational age; SYN1, syncytin-1; SYN2, syncytin-2.

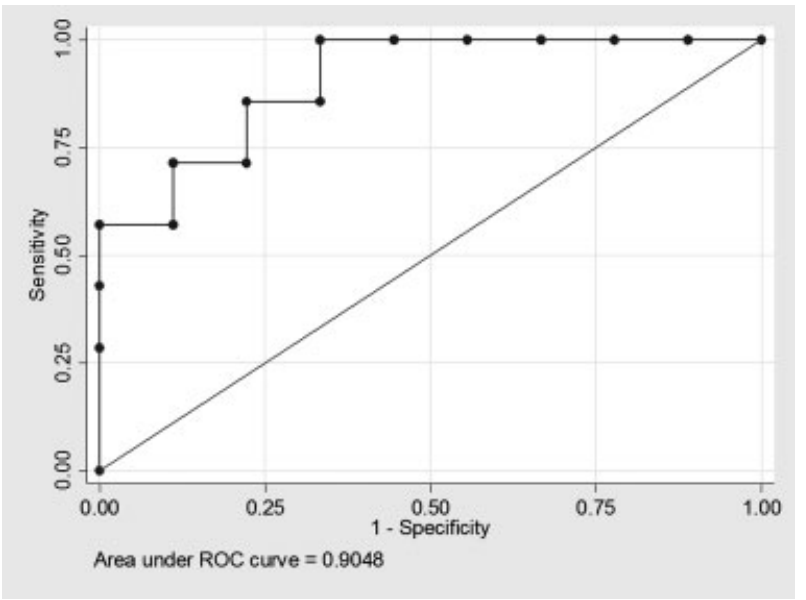

Fig. 2 Receiver-operating characteristic (ROC) curve of SYN1 + SYN2 placental methylation for the identification of FGR among FGR and SGA fetuses. FGR, fetal growth restriction; ROC, receiver-operating characteristic; SGA, small for gestational age.

FGR compared with SGA pregnancies. These differences could be used to distinguish pathologic FGR from constitutional SGA with reasonable predictive accuracy in this cohort. The methylation differences corresponded with a biologically consistent increase in expression of SYN1, though not SYN2. While DNA methylation regulates expression of both SYN1 and SYN2, expression patterns do not always follow that predicted by changes in DNA methylation. $^{22}$ This suggests that other mechanism regulate the expression of these critical gene products.

The methylation differences described in this study are consistent with previously published data showing differences in expression and methylation of these genes in growth discordant twins ${ }^{10}$ as well as pregnancies complicated by SGA ${ }^{11}$ and other placental syndromes. ${ }^{12-14}$ To our knowledge, however, this is the first study to attempt to assess differences in placental expression and methylation of SYN1 and SYN2 in pathologic FGR compared with physiologic SGA. This is of particular interest because it is biologically plausible that epigenetic marks such as DNA methylation are modifiable by environmental differences, such as hypoxia, that would lead to FGR versus SGA. An additional strength of our study is that all samples were obtained from deliveries done at 36 weeks or greater. This minimized the impact of gestational age on our results.

There are also limitations with the current study that deserve comment. First, the FGR and SGA groups were not identified prospectively and thus these groups may have 
some overlap. Nevertheless, the FGR group clearly represents a sicker population. Birth weights are smaller, there is a trend toward lower cord $\mathrm{pH}$ and a markedly increased rate of cesarean delivery compared with the SGA group. An additional concern is that our sample size in each group was small. To be more confident about the significance and magnitude of differences, larger studies replicating these results would be important. Additionally, by limiting our samples to term or near term, we may have missed cases with more severe FGR, as these would have been more likely to be delivered significantly preterm. As true differences would likely be exaggerated in more severely affected pregnancies, this decision should support the null hypothesis in the current study, but we cannot exclude the possibility of a more complicated relationship between SYN1 and SYN2 methylation and severity of placental dysfunction.

Despite these limitations, our data suggest that SYN1 and SYN2 may be useful biomarkers for distinguishing FGR from SGA. An important next step is a prospective study. To make these data clinically useful a source of placental DNA must be available prior to delivery. Thus, we propose to replicate our results in prospectively obtained cell-free fetal DNA, as the majority of this is suspected to be placental in origin. ${ }^{23}$ Studies have already been performed that illustrate the feasibility of assessing differential $\mathrm{CpG}$ methylation in cellfree fetal DNA and maternal DNA, ${ }^{24}$ supporting the potential of this approach.

In conclusion, we identified significant differences in methylation patterns of SYN1 and SYN2 that distinguished FGR from SGA. This work adds to a growing effort to define FGR biologically, rather than by a threshold centile on a growth curve. ${ }^{25}$ If these methylation differences are replicated in cell-free DNA, this approach has the potential to provide noninvasive information about placental function that could be used clinically.

\section{Presentation Information}

Findings presented at the 36th Annual Pregnancy Meeting, Society for Maternal Fetal Medicine, Atlanta, Georgia, February 1-6, 2016

\section{Funding Source}

This work was supported by the Reproductive Scientist Development Program grant NIH K12 HD000849 (K.P.H.) and National Institutes of Health Grants K12 HD063087 (K.P.H.)

\section{Conflict of Interest and Financial Disclosure}

The authors have no financial disclosures or potential conflict of interest.

\section{Acknowledgments}

We would like to acknowledge Dr. Yoel Sadovsky for contributing biological samples and for his mentorship and guidance throughout this study. In addition, we would like to posthumously thank Nicole Kotchey for her incredible organization and assistance in the initial stages of this project before her untimely passing.

\section{References}

1 American College of Obstetricians and Gynecologists. ACOG Practice bulletin no. 134: fetal growth restriction. Obstet Gynecol 2013;121(05):1122-1133

2 Dudley NJ. A systematic review of the ultrasound estimation of fetalweight. Ultrasound Obstet Gynecol 2005;25(01):80-89

3 Gifford WD, Pfaff SL, Macfarlan TS. Transposable elements as genetic regulatory substrates in early development. Trends Cell Biol 2013;23(05):218-226

4 Friedli M, Turelli P, Kapopoulou A, et al. Loss of transcriptional control over endogenous retroelements during reprogramming to pluripotency. Genome Res 2014;24(08):1251-1259

5 Rawn SM, Cross JC. The evolution, regulation, and function of placenta-specific genes. Annu Rev Cell Dev Biol 2008;24:159-181

6 Reiss D, Zhang Y, Mager DL. Widely variable endogenous retroviral methylation levels in human placenta. Nucleic Acids Res 2007;35(14):4743-4754

7 Muir A, Lever A, Moffett A. Expression and functions of human endogenous retroviruses in the placenta: an update. Placenta 2004;25(Suppl A):S16-S25

8 Weiss RA, Stoye JP. Virology. Our viral inheritance. Science 2013; 340(6134):820-821

9 Dupressoir A, Lavialle C, Heidmann T. From ancestral infectious retroviruses to bona fide cellular genes: role of the captured syncytins in placentation. Placenta 2012;33(09):663-671

10 Gao Y, He Z, Wang Z, et al. Increased expression and altered methylation of HERVWE1 in the human placentas of smaller fetuses from monozygotic, dichorionic, discordant twins. PLoS One 2012;7(03):e33503

11 Ruebner M, Strissel PL, Langbein M, et al. Impaired cell fusion and differentiation in placentae from patients with intrauterine growth restriction correlate with reduced levels of HERV envelope genes. J Mol Med (Berl) 2010;88(11):1143-1156

12 Langbein M, Strick R, Strissel PL, et al. Impaired cytotrophoblast cell-cell fusion is associated with reduced syncytin and increased apoptosis in patients with placental dysfunction. Mol Reprod Dev 2008;75(01):175-183

13 Kudaka W, Oda T, Jinno Y, Yoshimi N, Aoki Y. Cellular localization of placenta-specific human endogenous retrovirus (HERV) transcripts and their possible implication in pregnancy-induced hypertension. Placenta 2008;29(03):282-289

14 Ruebner M, Strissel PL, Ekici AB, et al. Reduced syncytin-1 expression levels in placental syndromes correlates with epigenetic hypermethylation of the ERVW-1 promoter region. PLoS One 2013;8(02):e56145

15 Alexander GR, Himes JH, Kaufman RB, Mor J, Kogan M. A United States national reference for fetal growth. Obstet Gynecol 1996;87 (02):163-168

16 Gimenez J, Montgiraud C, Oriol G, et al. Comparative methylation of ERVWE1/syncytin-1 and other human endogenous retrovirus LTRs in placenta tissues. DNA Res 2009;16(04):195-211

17 Skaar DA, Li Y, Bernal AJ, Hoyo C, Murphy SK, Jirtle RL. The human imprintome: regulatory mechanisms, methods of ascertainment, and roles in disease susceptibility. ILAR J 2012;53(34):341-358

18 Kainz B, Shehata M, Bilban M, et al. Overexpression of the paternally expressed gene 10 (PEG10) from the imprinted locus on chromosome 7q21 in high-risk B-cell chronic lymphocytic leukemia. Int J Cancer 2007;121(09):1984-1993

19 Schulte AM, Lai S, Kurtz A, Czubayko F, Riegel AT, Wellstein A. Human trophoblast and choriocarcinoma expression of the growth factor pleiotrophin attributable to germ-line insertion of an endogenous retrovirus. Proc Natl Acad Sci U S A 1996;93(25):14759-14764

20 Liang CY, Wang LJ, Chen CP, Chen LF, Chen YH, Chen H. GCM1 regulation of the expression of syncytin 2 and its cognate receptor MFSD2A in human placenta. Biol Reprod 2010;83(03):387-395

21 Ehrich M, Nelson MR, Stanssens P, et al. Quantitative highthroughput analysis of DNA methylation patterns by base- 
e24 Syncytin-1 and 2 Gene Methylation in Growth Restriction

specific cleavage and mass spectrometry. Proc Natl Acad Sci U S A 2005;102(44):15785-15790

22 Himes KP, Koppes E, Chaillet JR. Generalized disruption of inherited genomic imprints leads to wide-ranging placental defects and dysregulated fetal growth. Dev Biol 2013;373(01):72-82

23 Bianchi DW. Circulating fetal DNA: its origin and diagnostic potential-a review. Placenta 2004;25(Suppl A):S93-S101
Makaroun, Himes

24 Hatt L, Aagaard MM, Graakjaer J, et al. Microarray-based analysis of methylation status of CpGs in placental DNA and maternal blood DNA: potential new epigenetic biomarkers for cell free fetal DNA-based diagnosis. PLoS One 2015;10(07):e0128918

25 Benton SJ, McCowan LM, Heazell AE, et al. Placental growth factor as a marker of fetal growth restriction caused by placental dysfunction. Placenta 2016;42:1-8 